\title{
The Patient-Centered Medical Home Movement- Promise and Peril for Family Medicine
}

\author{
John C. Rogers, MD, MPH, MEd
}

"Strengthen the Core and Stimulate Progress: Assembling Patient-Centered Medical Homes" was the theme of my year as the Society of Teachers of Family Medicine President for 2007 to 2008. I advocated strongly for the patient-centered medical home (PCMH), especially its relevance to the educational experiences in our teaching practices. ${ }^{1-7}$ Realizing the risk of being accused of "flipflopping" on this issue, I do not think it would be prudent to continue to enthusiastically embrace this movement without some reflection and analysis. Although it is not a novel approach, I think an analysis of strengths, weaknesses, opportunities, and threats is in order. Here I will review briefly what I consider family medicine's internal strengths and weakness related to the PCMH and the external opportunities and threats the PCMH movement presents to family medicine. I will end with a general action agenda.

\section{Strengths}

It helps me to divide the Joint Principles of the Patient-Centered Medical Home ${ }^{8}$ into 2 categories: (1) the type of care we provide to patients and (2) the practice infrastructure that supports our work. ${ }^{3}$ The "care principles" are the personal physician, physician-directed medical practice, whole person orientation, and that care is coordinated

Submitted 8 July 2008; revised 8 July 2008; accepted 8 July 2008.

From the Department of Family and Community Medicine, Baylor College of Medicine, Houston, TX.

Funding: none.

Conflict of interest: none declared.

Corresponding author: John C. Rogers, MD, MPH, MEd, Baylor College of Medicine, Family and Community Medicine, 3701 Kirby Dr., Suite 640, Houston, TX 77098 (Email: jrogers@bcm.edu).

See Related Articles on Pages 427, 441, 451, and 458. and/or integrated. The "infrastructure principles" are that care is coordinated and/or integrated, quality and safety, enhanced access, and payment for added value. Note that coordination and integration of care is a care principle in that we strive to do this for individual patients, but it is an infrastructure principle when it requires registries, information technology, and health information exchange.

We have been striving and succeeding for decades to practice in ways that are consistent with the care principles. We also have a long history of teaching students and residents about this type of care with accreditation standards that enforce achievement of the principles in resident training. We also have considerable scholarship and research documenting our provision of this care and its value to patients and community health, as illustrated in part by Rosenthal's article in this issue of the fournal of the American Board of Family Medicine.

Our strength with the infrastructure principles is our commitment to evidence-based clinical guidelines, quality improvement, and information technology and our efforts to apply these to our routine medical care. We have well-developed approaches for bringing the best information to the point of care and have growing experience with enhanced access through open scheduling, expanded hours, and electronic communication.

There is also strength in understanding the process of change in clinical practices and how to facilitate that process. We have a solid scholarship base in this area and practical experience with the TransforMED and $\mathrm{P}^{4}$ projects. ${ }^{10}$ Strengths in the care and infrastructure principles and the change process are a foundation for further progress.

\section{Weaknesses}

One of our weaknesses regarding the care principles is less than universal provision of desired communication skills and shared decision making, as observed by DeVoe et al in this issue of the fournal 
of the American Board of Family Medicine. ${ }^{11}$ Another is that, although we may be providing continuous, team-based care that meets many patients' health needs, we do not have systems that allow us to readily document (1) that each patient has an ongoing relationship with a personal physician trained to provide first contact and continuous and comprehensive care (personal physician); (2) that a personal physician leads a team of individuals at the practice level who collectively take responsibility for the ongoing care of patients (physician-directed medical practice); (3) that a personal physician is responsible for providing for all of the patient's health care needs or takes responsibility for appropriately arranging care with other qualified professionals (whole person orientation); and (4) that care is coordinated and/or integrated across all elements of the complex health care system and the patient's community. ${ }^{1}$ Furthermore, although we believe in patient involvement in care, few of us are in practices that can boast that we use a formal care planning process between physicians and patients, that we regularly seek feedback to ensure patients' expectations are being met, and that we involve patients and families in quality improvement activities at the practice level (quality and safety principle).

These deficiencies underscore the fact that our greatest weakness is in the infrastructure principles. We have few practices that have adequate resources (capital and time) for these other elements of the care coordination and quality and safety principles:

- registries, information technology, health information exchange;

- evidence-based medicine and clinical decisionsupport tools;

- continuous quality improvement through performance measurement and improvement;

- information technology to support patient care, performance measurement, patient education, and enhanced communication; and

- demonstration that the practice has the capabilities to provide patient-centered services consistent with the medical home model.

The TransforMED National Demonstration Project has identified the difficulties in changing practices to become patient-centered medical homes:

"Creating a PCMH is much more than a sum of implementing discrete model components.
Such transformation is exceedingly difficult, and those who attempt it are heroic. To achieve transformation, full engagement of critical members of the practice is needed. At the same time the practice needs to remain in charge of its own destiny. They may need assistance in making the changes, but the decision what to change needs to be theirs. They also need to remain full partners in their learning and development process."12

Our weaknesses in the change process include the paucity of resources-time and money-to support practice transformation, the limited diffusion of the knowledge and skills necessary for change management, and where many of us are in the stages of change related to the PCMH (precontemplation or contemplation). Family medicine is rather weak on being able to document the care it provides, supporting the cost and effort of implementing the infrastructure elements in the PCMH principles, and supporting and managing the change process.

\section{Opportunities}

The PCMH movement may bring recognition, reward, and support to family medicine. Contrary to the negative recognition we received in the HMO/ managed care movement as gatekeepers who were to control access to other providers and services, we are now receiving more positive recognition as gateways $^{13}$ whose role is to coordinate and integrate access. This time the recognition seems to be accompanied by an acknowledgment of both the health and financial benefits to patients and communities of an available primary care base. It is our intrinsic worth-just being present-that benefits the public, not our control of access to other services. The reward and support may be increased compensation to individual physicians and reimbursement that will fund development of infrastructure provided that the payment system fulfills the stated principles:

- It should pay for services associated with coordination of care.

- It should allow for separate fee-for-service payments.

- It should allow for additional payments for achieving measurable quality improvements. 
- It should support provision of enhanced communication access.

- It should support use of health information technology for quality improvement.

Recognition, reward, and support are sometimes viewed in terms of power and prestige; an alternative view is to think of them in terms of equality and equity. Either way, more appropriate levels may help with retention in practice of current family physicians and enhance the recruitment of students to family medicine that would be necessary to meet the goal of a personal medical home for every person in the United States.

\section{Threats}

The opportunity for enhanced reimbursement will probably depend on the principle that practices go through a voluntary recognition process by an appropriate nongovernmental entity to demonstrate that they have the capabilities to provide patientcentered services consistent with the medical home model. ${ }^{1}$

The National Committee for Quality Assurance's (NCQA) Physician Practice Connections Patient-Centered Medical Home (PPC-PCMH) Recognition Program ${ }^{14}$ will be prominent. The TransforMED Medical Home $\mathrm{IQ}^{10}$ instrument shows practices how they would score on the NCQA PPC-PCMH Recognition Program standards and identifies explicit changes necessary to achieve that recognition. The threat is that the recognition criteria emphasize infrastructure principles and provide little, if any weight, to care principles. This may distort what is perceived as important in the PCMH movement. Focusing mostly on implementing the infrastructure principles is a risk:

"Early analysis from the NDP [National Demonstration Project] (using both qualitative data and the quantitative scores from 2 waves of patient outcome surveys) does suggest that implementing components of the original TransforMED model does not automatically lead to a patient-centered medical home. A primary focus of the NDP has been implementation of the TransforMED model components and to a certain extent, this focus on technical innovations has competed with efforts to address re- lationship-centered patient care within the practice."

"Successful implementation of new model components does not automatically lead to the relationship-centered organization, necessary for sustained change and learning. This is understandable, as the hard work of implementing technology and revamping workflow means the difficult task of building practice relationships retreat to the backburner. For the most part, the practices that are relationship-centered were so in the beginning." 12

The threat to family medicine is that a recognition program will emphasize infrastructure and forget that relationship-centered care is the core of the $\mathrm{PCMH}$ and that the purpose of infrastructure is to support patient-centered care, not divert from it.

The Patient-Centered Primary Care Collaborative ${ }^{8}$ has approximately 150 members representing every major stakeholder group in medical care, including physician associations, health systems, patient organizations, insurance companies, and business groups. A prominent motivation for many of these groups advocating for the PCMH is the payment principle: "Payment appropriately recognizes the added value provided to patients who have a patient-centered medical home." ${ }^{1}$ For patient organizations, "added value" seems to mean improved quality of care, for insurance companies it seems to mean cost savings, and for businesses it seems to mean more care for less money. This "value" emphasis holds several threats for us, as raised by Fink in this issue of the fournal. ${ }^{15}$ One threat to family medicine is how quality of care is measured. Any family medicine faculty member who has participated in quality improvement efforts knows all too well that the indicators of quality and the data systems for collecting these data can be flawed. Further, individual practices could be penalized if the principle about payment ("It should recognize case mix differences in the patient population being treated within the practice."1) is ignored and "cherry-picking" of patients is allowed. A threat, and inequity, is if the insurance companies ignore a powerful principle- "It should allow physicians to share in savings from reduced hospitalizations associated with physician-guided care management in the office setting." ——and pocket rather than share the savings that family physicians create for them. Another threat is if these stakeholders fail 
to realize that the PCMH is not a panacea for the rising costs of health care and abandon the $\mathrm{PCMH}$ concept when health care costs continue to rise and lead to continuing increases in insurance premiums for businesses.

I may be cynical, but I know that one threat to family medicine will be the PCMH bandwagon where wannabes, lookalikes, and opportunists put out "PCMH" shingles claiming they are a "medical home" and deserve the financial rewards intended for the thousands of family physicians throughout this country who have been unsung real medical homes for decades. The core principles of the PCMH must be upheld to prevent it from becoming just another modification of disease management or carve-out programs.

A most dangerous threat to family medicine will be those who co-op the vision of the PCMH and distort the real meaning of what constitutes a medical home. Our vision, and the vision our leaders have been articulating for just shy of 40 years, is still in many ways a counter-culture vision of relationship-centered, patient-centered, family-centered care that integrates behavioral science into the fabric of our continuing, comprehensive care of patients and families. This is the vision that will realize the promise that the PCMH holds for the health and well-being of our patients and communities.

The PCMH movement may threaten family medicine by the emphasis of the recognition programs on infrastructure, the value orientation focus on cost savings, PCMH imposters, and the possibility of a stolen vision. These threats must not be minimized or ignored. Our general tendency in family medicine is to be modest and to assume benign intent on the part of others. This is fundamental to the power of our patient-centered, family-centered, relationship-centered care. But to counter the real threats to the PCMH and our field, we must be alert, diligent, and skeptical. Our advocacy is not self-centered but for the benefit of our patients and communities whose health and wellbeing is enhanced by our existence.

\section{Action Agenda}

I suggest a simple yet difficult and demanding action agenda.

\section{Clinical Care}

We move swiftly with wise, reasoned, and paced change toward the PCMH principles in every one of our clinical practices. All of us must become change agents advocating for advances in the care we provide. We must regularly measure our practices against the external benchmarks provided by the TransforMED Medical Home IQ and NCQA PPC-PCMH Recognition Program.

\section{Research}

We quickly identify the key questions regarding the effectiveness and implementation of the PCMH principles. We must determine what is effective and how to achieve it.

\section{Education}

We specify the teaching practices and teaching methods necessary to prepare students, residents, and faculty for practice in PCMHs. Given vary rapidly evolving medical care and the $\mathrm{PCMH}$ model, we also must identify the best methods for rapid just-in-time skill acquisition.

\section{Advocacy}

We clearly, consistently, continuously and loudly articulate our vision for patient-centered, familycentered, relationship-centered care. We must stay "on message" about the PCMH. We must not let others co-op the PCMH concept. We must never forget, or let others forget, that care is what we do, and infrastructure supports that role.

\section{Conclusion}

The PCMH movement offers us a defining moment in the development, evolution, and even survival of our field. I think this opportunity blends our core values of what we do in clinical care, our beliefs about equity and justice in health care, and our self-image as counter-culture and change agents. Forty years ago some visionaries saw clearly the needs of their patients and communities and said there is a better way. ${ }^{16}$ Today many other voices have joined our ancestors to say that medical care can be better, it must be better. Let us honor our past, stand strong for our beliefs, and help the system see the wisdom of the PCMH and a strong primary care base, for the benefit of our patients and our communities. 


\section{References}

1. Rogers J. Strengthen the core and stimulate progress: assembling patient-centered medical homes. Fam Med 2007;39:465-8.

2. Rogers J. Assembling patient-centered medical homes-is this focus on patient care a distraction from STFM's primary mission? Fam Med 2007;39:542-3.

3. Rogers J. Assembling patient-centered medical homes-the care principles. Fam Med 2007;39:697-9.

4. Rogers J. Assembling patient-centered medical homes-the promise and price of the infrastructure principles. Fam Med 2008;40:11-2.

5. Rogers J. Assembling patient-centered medical homes-teaching resources. Fam Med 2008;40:85-6.

6. Rogers J. Assembling patient-centered medical homes-the clerkship initiative. Fam Med 2008;40: $163-4$.

7. Rogers J. Assembling patient-centered medical homes in teaching practices- one strategy. Fam Med 2008;40:237-40.

8. Patient-Centered Primary Care Collaborative. [Homepage.] Available at http://www.pcpcc.net. Accessed 8 July 2008.

9. Rosenthal TC. The medical home: growing evidence to support a new approach to primary care. J Am Board Fam Med 2008;21:427-40.
10. TransforMED. [Homepage.] Available at http://www. transformed.com. Accessed 8 July 2008.

11. DeVoe JE, Wallace LS, Pandhi N, Solotaroff R, Fryer GE. Comprehending care in a medical home: a usual source of care and patient perceptions about healthcare communication. J Am Board Fam Med 2008;21:441-50.

12. Stewart E, Jaén C, Crabtreee B, Nutting P, Miller W, Stange K. Evaluators' report on the National Demonstration Project (NDP) to the board of directors of TransforMED. TransforMED. Available at http://www.transformed.com/evaluatorsReports/ report5.cfm. Accessed 8 July 2008.

13. Daaleman TP. The medical home: locus of physician formation. J Am Board Fam Med 2008;21:451-7.

14. National Committee for Quality Assurance. Physician Practice Connections-Patient-Centered Medical Home (PPC-PCMH). Available at http:// web.ncqa.org/tabid/631/Default.aspx. Accessed 8 July 2008.

15. Fink KS. Value driven health care: proceed with caution. J Am Board Fam Med 2008;21:458-60.

16. Taylor R. The promise of family medicine: history, leadership, and the age of aquarius. J Am Board Fam Med 2006;19:183-90. 